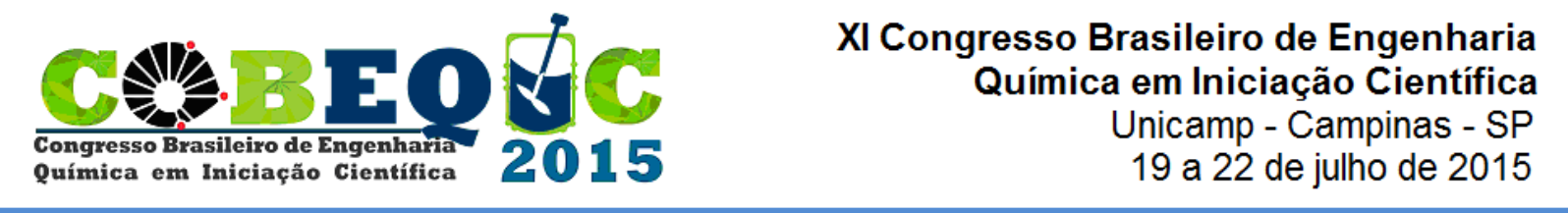

\title{
Inventário da para disponibilidade de Resíduos Sólidos Urbanos para fins de matéria prima para os setores energético e químico. Região Administrativa de Campinas - São Paulo.
}

\author{
F. B. CASAGRANDE ${ }^{1,2}$, M. A. ARAUJO ${ }^{1,2}$, T. T. FRANCO ${ }^{1,2}$, R. LAMPARELLI ${ }^{1}$, M. \\ BERNI ${ }^{1}$ \\ ${ }^{1}$ Universidade Estadual de Campinas, Núcleo Interdisciplinar de Planejamento Energético. \\ ${ }^{2}$ Universidade Estadual de Campinas, Faculdade de Engenharia Química. \\ ${ }^{3}$ Universidade Estadual de Campinas, Faculdade de Engenharia Mecânica.
}

\section{E-mail para contato: fernandacasagrande@nipeunicamp.org.br}

RESUMO - Este projeto tem por objetivos principal desenvolver e implantar metodologias para inventariar e montar bancos de dados georreferenciado de resíduos sólido urbano RSU das Região Administrativa (RA) de Campinas (São Paulo). Após implementação e teste das metodologias, um projeto ampliado para estimar e integrar localização, qualidade, quantidade, sazonalidade, entre outros deverá ser submetido em Projeto Temático à Fapesp, ampliando o escopo (maior numero de regiões administrativas e com maior participação de interessados/stakeholders). O inventário e o banco de dados gerados também poderão ser utilizados em intermediações com os produtores de bioenergia e de matéria-prima, potenciais clientes interessados, bem como colaborar com a elaboração de novas políticas públicas, tomada de decisões de investimento e preparo de legislação para estimular o direcionamento dos resíduos. Este projeto irá estudar a RA de Campinas; levantando dados e demonstrando a janela de oportunidades para o aproveitamento de resíduos sólidos urbanos. 


\section{INTRODUÇÃO}

Preocupações ambientais associadas à recente crise econômica aumentaram ainda mais a necessidade de inovações pelo setor industrial na busca de maior competitividade e crescimento econômico. A parcial substituição de recursos fósseis para obtenção de novos compostos químicos, energia e combustíveis pode ser alcançada com eficiente utilização de recursos renováveis, bem como pelo aumento uso de matérias consideradas "resíduos" agrícolas, agroindústrias, florestais e sólido urbano (RSU). (Lima et al., 2011; Lucke, 2012; Mamede, 2012; Foster-Carneiro et al., 2013; e Ki Lin, et al., 2014). O tema aqui proposto trata da primeira etapa metodológica de Projeto Temático. Tratamos de investigar a disponibilidade de resíduo sólido urbano (RSU) para geração de Bioenergia e Bioprodutos e comparar com as possíveis demandas dos potenciais clientes (indústrias químicas e de energia). Também avalia a infraestrutura disponível para comercialização e distribuição dos potenciais produtos de origem renovável gerados, exemplo os blocos construtores, energia e combustíveis.

\section{DESAFIOS DO PROJETO}

Dois grandes temas integrados constituem o foco principal deste projeto de pesquisa, Grande quantidade de resíduos gerados no Estado de São Paulo associada à escassez de energia e de materiais (compostos químicos) de baixo impacto e de origem renovável.

O Estado de São Paulo e seus 645 municípios produziram em 2013 a quantidade de 59,291 toneladas /dia de RSU, $99 \%$ foram coletadas. Os dados indicam crescimento de 4,9\% no total coletado e aumento de 4,7 \% na geração de RSU em relação ao ano anterior. Em 2013, cerca de 23,6 \% do RSU do estado (correspondentes a 13,865 toneladas diárias) foram destinados aos "lixões" e aterros controlados, os quais do ponto de vista ambiental, pouco se diferenciam dos próprios lixões, pois não possuem o conjunto de sistemas necessários para proteção do meio ambiente e da saúde pública ambientais. (ABRELPE, 2014).

O RSU pode ser utilizado para melhorar condições físicas e químicas do solo, como fertilizantes naturais. Assim, o uso de composto de "lixo urbano" na "formulação de substratos para o cultivo de plantas em recipientes contribui significativamente para a melhoria da fertilidade do substrato", à medida que o composto de RSU apresenta grandes quantidades de compostos nitrogenados, (Lima et al 2010).

Mamede (2013) em sua dissertação descreve os principais meios de aproveitamento energético para atendar a demanda crescente de energia, assim como normas estabelecidas e políticas vigentes nos âmbitos socioeconômico e ambiental. A separação do lixo em seus componentes mais brutos: lixo orgânico, reciclável e rejeitos em geral, permite rotas diferentes de aproveitamento das diferentes frações: digestão anaeróbica para orgânicos, produção de "combustíveis derivados de resíduos" (CDR) para resíduos digeridos e incineração de rejeitos orgânicos e não orgânicos conforme a Figura 1.

Segundo o relatório [r]evolução energética (Krewitt et al., 2007) numa análise de evolução, considerando o ano de 2003 até 2050 constatou que haverá aumento de eficiência nos sistemas energéticos assim como uma evolução na utilização de energias não renováveis. Mas há ainda muitos obstáculos a serem vencidos e estudados. Uma das questões a ser melhor avaliada é se a energia deve ser concentrada ou descentralizada. Fora outras questões a resposta para esta pergunta deve estar atrelada a uma importante informação de apoio, isto é, a sua localização. A mais promissora e consolidada ferramenta de tratamento de dados espaciais é o Sistema de 
Informação Espacial (SIG). Essa ferramenta tem a capacidade de analisar fenômenos espacialmente distribuídos através das variáveis que o governam.

Figura 1 - Reaproveitamento do Resíduo Sólido Urbano segundo (Mamede, 2013)

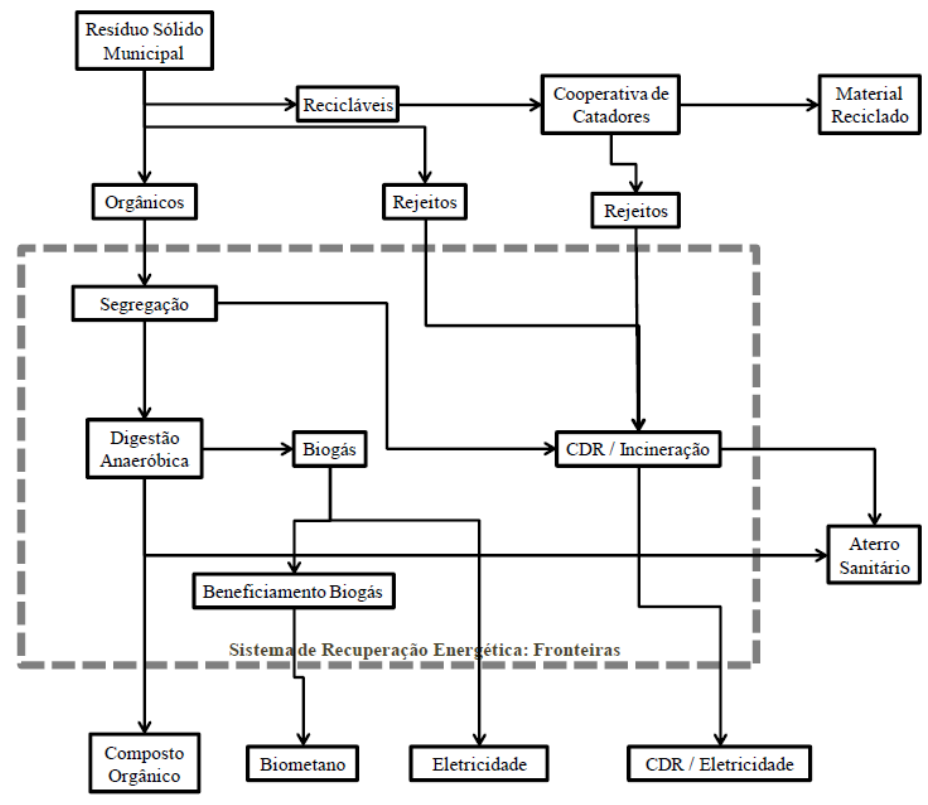

\section{METODOLOGIA:}

\subsection{Data Mining}

O Data Mining (expressão inglesa) consiste em nossa primeira parte do Projeto Temático o qual irá buscar, organizar e associar dados na finalidade de obter uma agregação de valor no final. Um exemplo disso está encontrado na tabela 1 extraída do Relatório Estadual de Resíduos Sólidos Urbanos CETESB do abaixo: 
Tabela 1: Quadro Geral da Região Metropolitana de Campinas.

\begin{tabular}{|c|c|c|}
\hline \multirow[b]{2}{*}{ Cidade } & \multicolumn{2}{|c|}{ Região Metropolitana De Campinas } \\
\hline & População & Lixo (t/dia) \\
\hline Americana & 210.638 & 201,15 \\
\hline Artur Nogueira & 44.177 & 35,07 \\
\hline Campinas & 1.080 .113 & 1237,69 \\
\hline Cosmópolis & 58.827 & 47,86 \\
\hline Engenheiro Coelho & 15.721 & 9,05 \\
\hline Holambra & 11.299 & 6,44 \\
\hline Hortolândia & 192.692 & 188,23 \\
\hline Indaiatuba & 201.619 & 197,83 \\
\hline Itatiba & 101.471 & 74,23 \\
\hline Jaguariúna & 44.311 & 38,46 \\
\hline Monte Mor & 48.949 & 40,19 \\
\hline Morungaba & 11.769 & 7,55 \\
\hline Nova Odessa & 51.242 & 43,46 \\
\hline Paulínia & 82.146 & 74,07 \\
\hline Pedreira & 41.558 & 35,31 \\
\hline Santa Bárbara d'Oeste & 180.009 & 100,14 \\
\hline Santo Antônio de Posse & 20.650 & 14,02 \\
\hline Sumaré & 241.311 & 229,96 \\
\hline Valinhos & 106.793 & 99,61 \\
\hline Vinhedo & 63.611 & 54,21 \\
\hline
\end{tabular}

Com conhecimento desse quadro geral podemos ilustrar, com a entrevista do Doutor Sérgio Augusto Lucke para o Jornal da Unicamp, o qual relatou que se o lixo da cidade de Campinas fosse incinerado haveria cerca de $15 \%$ a mais de energia elétrica em relação a consumida, caso contasse com uma usina para incinerar suas 1.050 toneladas diárias de lixo. Considerando que a margem de segurança de risco de apagão é de 3 a 5\%, 15\% ofereceria muita segurança. (Jornal da Unicamp)

\subsection{Sistema de Georreferenciamento (Municípios, RSU, Sistema de Transporte)}

A análise espacial de fenômenos na superfície da Terra deve obedecer a uma regra comum, a possibilidade de ser georreferenciado a um sistema de referência. Assumindo que isso seja possível, duas etapas para análise do fenômeno são necessárias: primeiramente, levantar dados relacionados as variáveis desejadas, através do Sistema de Posicionamento Global (GPS) e Sensoriamento Remoto (SR); e em segundo, análise dos dados por Sistemas de Informações Geográficas (SIG).

Os dados levantados serão complementados em um Banco de Dados da RA de Campinas, coletados via GPS e SR contendo variáveis de logística (estradas, rios, estradas de ferro), de influência (aterros próximos, empresas, armazéns) e populacional. Para isso, utilizaremos como material os recursos: 
Material de Base:

- Imagens de satélite Landsat 8 (Resolução espacial 30 × 30 m/ 100 × 100 m, res. temporal 16 dias, res. radiométrica 16 bits e res. espectral 9 bandas);

- Imagens de satélites Rapideye (Resolução espacial $5 \mathrm{~m}$, res. temporal 1 dia, res. radiométrica 16 bits e res. espectral 5 bandas);

- Dados de Infraestrutura obtidos da CONAB (Companhia de Abastecimento do Ministério da Agricultura e Pecuária), e do MMA (Ministério do Meio Ambiente);

- Dados de população IBGE (Instituto Brasileiro de Geografia e Estatística);

- Dados de geração de RSU obtidos da CETESB (Companhia de Tecnologia de Saneamento Ambiental);

Além disso, os dados espaciais coletados estarão integrados aos softwares ArcGis ou na plataforma do Google Maps, na finalidade de obter uma melhor visualização dos dados georreferenciados.

Já a análise dos Sistemas de Informações Geográficas (SIG) incorpora um sofisticado conjunto de procedimentos computacionais baseado em algoritmos para trabalhar com dados espaciais (Cowen, 1990). Esse trata com o fluxo de dados e informação a partir de sua fonte primária para uma informação derivada ou uso final. Portanto essa análise pode investigar a disponibilidade de resíduo sólido urbano (RSU) para geração de bioprodutos (incluindo bioenergia) e comparar demandas dos potenciais clientes.

\section{CONCLUSÕES:}

Esse trabalho apresenta a finalidade de mostrar o papel da análise espacial em conjunto da gestão dos Resíduos Sólidos Urbanos. Aplicou conceitos, técnicas e procedimentos inerentes ao geoprocessamento e análise espacial. Um caso específico, deverão ser propostas metodologias integradoras ao qual se diz respeito ao banco de dados, como que oferecem informações preliminares tal qual auxiliam o direcionamento no cumprimento das etapas da gestão dos resíduos.

Inventário e banco de dados georreferenciados dos RSU: irá permitir novos estudos econômicos, energéticos e ambientais na cadeia de suprimento do RSU por Região Administrativa do Estado de São Paulo.

A ideia é integrar os RSU, infraestrutura e demanda populacional e das indústrias químicas e de em energia dos respectivos RA, com a possível utilização dos mesmos como matéria prima para geração de produtos com aplicações diversas no mercado. 


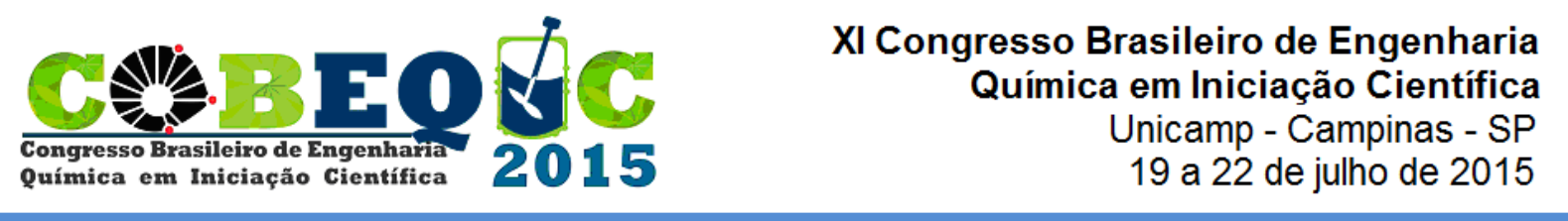

\section{NOMENCLATURA}

- CETESB: Companhia de Saneamento Ambiental

- Composto Químico: Qualquer composto químico, bioquímico, polímeros, bio-polímeros, blocos construtores, entre outros.

- GPS: Generalized System of Preferences

- IBGE: Instituto Brasileiro de Geografia e Estatística

- IEA: Instituto de Economia Agrícola

- RA: Região Administrativa.

- Resíduo Agrícola: Resíduo que fica no campo após colheita do produto principal, incluindo resíduos de florestas plantadas.

- Resíduo Agroindustrial: Resíduo vegetal já transportado à agroindústria juntamente com a parte principal da planta a ser processada, incluindo aqueles do setor de florestas plantadas, que necessita ser tratada para disponibilização final.

- Resíduo Sólido Urbano (RSU): Resíduo urbano coletado, previamente separado ou não.

- RPI: Índice de produção de resíduos (Residues production index)

- SEADE: Fundação sistema estadual de análise de dados

- SIG: Sistema de informação Geográfica

- SR: Sensoriamento Remoto

- UGRH: Unidade de Gerenciamento de Recursos Hídricos.

\section{REFERÊNCIAS}

ABRELPE, Associação Brasileira de Empresas de Limpeza Pública e Resíduos Especiais, "Panorama dos Resíduos Sólidos no Brasil 2013". Disponível in www.abrelpe.org.br, acessado em setembro 2014.

Cardoso, T.F; Otávio Cavalett ${ }^{1}$, Mateus Ferreira Chagas ${ }^{1}$, Edvaldo Rodrigo de Morais ${ }^{1}$, João Luís Nunes, Carvalho ${ }^{1}$, Henrique Coutinho Junqueira Franco ${ }^{1}$, Marcelo Valadares Galdos ${ }^{1}$, Fábio Vale Scarpare ${ }^{1}$, Oscar Antonio Braunbeck ${ }^{1}$,Luis Augusto Barbosa Cortez ${ }^{2}$, Antonio Bonomi ${ }^{1}$, “Technical and economic assessment of trash recovery in the sugarcane bioenergy", Sci. Agric. v.70, n.5, p.353-360, September/October 2013.

Cortez, L. A., "Roadmap for sustainable aviation biofuels for Brazil", Projeto Financiado Fapesp-Boeing-Embraer, Editora Blucher, 2014.

Dansereau, L.P., El-Halwagi, M., Chambost, V.; Stuart, P., "Methodology for biorefinery portfolio assessment using supply-chain fundamentals of bioproducts", Wiley Online Library; DOI: 10.1002/bbb.1490; Biofuels, Bioprod. Bioref. 8:716-727, 2014.

Dias, M., Tassia L. Junqueira, Otávio Cavalett a, Lucas G. Pavanello a, Marcelo P. Cunha, Charles D.F. Jesus a, Rubens Maciel Filho a,b, Antonio Bonomi a, "Biorefineries for the production of first and second generation ethanoland electricity from sugarcane". Applied Energy 109 (2013) 72-78.

Ensinas, A. V., "Estudo da geração de biogás no aterro sanitário Delta em Campinas" SP, Dissertação (mestrado), Universidade Estadual de Campinas, Faculdade de Engenharia Mecânica, UNICAMP, Campinas, SP, 2003.

EPE (Empresa de Pesquisa Energética), Série Recursos Energéticos, NOTA TÉCNICA DEA 18/14, "Inventário Energético dos Resíduos Sólidos Urbanos", pág. 12, Rio de Janeiro, Outubro, 2014. 
Faaij. A, IEA Roadmap Workshop "Sustainable feedstock supply for bioenergy and biofuels", Copernicus Institute, Faculty of Science, Utrecht University, Task Leader IEA Bioenergy Task 40, Paris - France, IEA HQ, 15-16 September 2010, Disponible in https://www.iea.org/media/bioenergyandbiofuels/07 Faaij.pdf, accessed, in october 2014.

Forster-Carneiro T., BERNI, M.D., Dorileo I.L., Rostagno, M.A., "Biorefinery study of availability of agriculture residues and wastes for integrated biorefineries in Brazil." In: Resources, Conservation and Recycling, Copyright (C) 2013 Elsevier B.V. All rights reserved, 5-Year Impact Factor: 2.889, ISSN: 0921-3449, Volume 77, August 2013, Pages 78-88. Disponible in http://www.sciencedirect.com/science/journal/09213449/77

Guanabara, R., Gama, T., Eigenheer, E. M.; "Contribuições para a construção de uma matriz para avaliação de projetos de educação ambiental”, Universidade Federal Fluminense, Mai/Ago. 2009.

IEA, Bioenergy Annual Report, 2011, 124 p.

IEA, Bioenergy Annual Report, 2013, 140 p.

IEA, International Energy Agency, ECONOMIC SUSTAINABILITY OF BIOMASS FEEDSTOCK SUPPLY, IEA BIOENERGY: Task 43: TR2013:01, Lead Authors: Tanja Ikonen (Finnish Forest Research Institute, Finland), Antti Asikainen (Finnish Forest Research Institute, Finland), accessed, September, 2014.

IPEA, Instituto de Pesquisa Econômica Aplicada, "Diagnóstico dos Resíduos Orgânicos do Setor Agrossilvopastoril e Agroindústrias Associadas", Relatório de Pesquisa, 2012, 134 p.

Itaú Sustentabilidade, disponível em http://ww2.itau.com.br/sustentabilidade/ /no-seu-dia-adia/sustentabilidade-na-pratica/cases/case-uteb.html acessado em novembro de 2014

Lima R. L. S., Severino L. S., Sofiatti V., Gheyi H. R.; “Atributos químicos de substrato de composto de lixo orgânico", 4 \& Nair H. C. Arriel, UAEA/UFCG- Campina Grande/PB-Brasil, 29/11/2010.

Lucke, S. A.; "O resíduo sólido urbano como fonte renovável para geração de energia elétrica: aspectos econômicos e sócio-ambientais.", Universidade Estadual de Campinas, Faculdade de Engenharia Civil, Arquitetura e Urbanismo, 2012

Ki Lin, C.S, Koutinas, A.A., Stamatelatou, K., Mubofu, E.B., Matharu, A.S., Kopsahelis, N., Pfaltzgraff, L.A., Clark, J.H., Papanikolaou, S., Kwan, T.H., Luque, R., "Current and future trends in food waste valorization for the production of chemicals, materials and fuels: a global perspective", Biofuels, Bioprod. Bioref. 8:686-715, Wiley Online Library DOI: 10.1002/bbb.1506; 2014.

Mamede, M. C. S., “Avaliação Econômica e Ambiental do Aproveitamento Energético de Resíduos Sólidos no Brasil”, Universidade Estadual de Campinas, Faculdade de Engenharia Mecânica, 2013.

Mantovani, V. A., “Caracterização detalhada dos Resíduos Sólidos Domiciliares de Sorocaba visando melhorias do sistema de coleta seletiva", Sorocaba, 2013.

Mourad, Anna L., Ambrogi, Vinicius S. and Guerra, Sinclair M. G. "Potencial de utilização energética de biomassa residual de grãos." In: ENCONTRO DE ENERGIA NO MEIO RURAL, 5, 2004, Campinas.Proceedings online. Available from: 


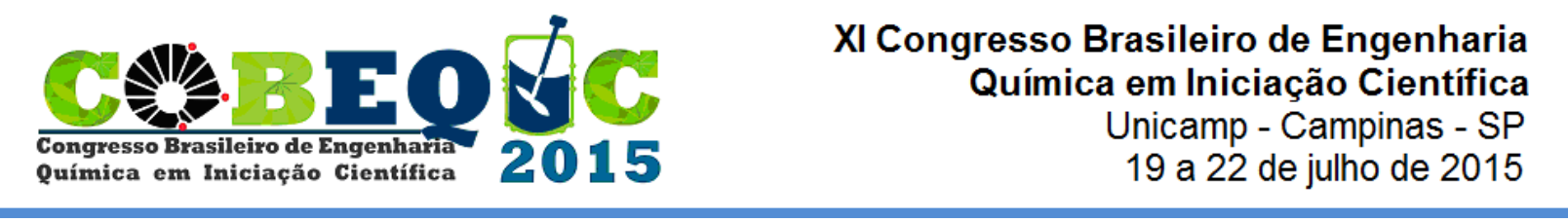

$<$ http://www.proceedings.scielo.br/scielo.php?script=sci_arttext\&pid=MSC00000000220040002000 33\&lng=en\&nrm=abn>. Acessed on: 17 Oct. 2014.

MMA, Ministério do Meio Ambiente, "Política Nacional de Resíduos Sólidos", acessado no site: http://www.mma.gov.br/pol\%C3\%ADtica-de-res\%C3\%ADduos-s\%C3\%B3lidos, acessado em 24/11/2014.

Oliveira, L. G. S., "Aproveitamento energético de resíduos agrícolas - $O$ caso da agroeletricidade distribuída”, Rio de Janeiro: UFRJ, COPPE, 2011. XIX, 282 p.

Scarlat, N., Dallemand, J., Banja, M., Possible impact of 2020 bioenergy targets on European Union land use. A scenario-based assessment from national renewable energy action plans proposals, Renewable and Sustainable Energy Reviews, Waste management 30, ELSEVIER, 2013, p. 18, 595-606.

Souza, R. A.; França, S. A. M.; "Lixo, Conduta Humana e a Gestão dos Insuportáveis", Universidade Estadual Paulista Júlio de Mesquita Filho, Faculdade de Ciências e Letras de Assis, 2013.

Souza, R. L. R., Regina, A.; Fontes, M., Salomão, S.: “A triagem de materiais recicláveis e as variabilidades inerentes ao processo: estudo de caso em uma cooperativa", 11/07/2014

Jornal da Unicamp “A usina que faz o lixo desaparecer", 04/03/2013

Relatório Estadual de Resíduos Sólidos Urbanos, CETESB,2013

Krewitt, W.; Simon, S.; Kronshage S.; Graus, W.; Harmelink, M.[r]evolução energéticaPerspectivas para uma Energia Global Sustentável. Relatório cenário brasileiro. EREC e Greenpeace. 2007. 99 p. (Consultado em 24/09/2013) http://www.greenpeace.org.br/energia/pdf/cenario brasileiro.pdf

Cowen, D.J. GIS versus CAD versus DBMS: what are the differences? Cap. 4. p. 52-61. In: Introductory readings in Geographic Information Systems. Ed. Peuquet e Marble. Taylor \& Francis. Bristol/England. 1990. 371 p. 